\title{
New Korean Record of Twenty Eight Species of the Family I chneumonidae (Hymenoptera)
}

\author{
Jin-Kyung Choi ${ }^{1}$, Jong-Chul Jeong ${ }^{2}$, Jong-Wook Lee ${ }^{1, *}$ \\ 'Department of Life Sciences, Yeungnam University, Gyeongsan 712-749, Korea \\ ${ }^{2}$ National Park Research Institute, Korea National Park Service, Namwon 590-811, Korea
}

\begin{abstract}
We report twenty eight ichneumonid species new to Korea. These species belong to seven subfamilies. Among them five subfamilies, Diacritinae Townes, 1965, Microleptinae Townes, 1958, Orthocentrinae Förster, 1869, Orthopelmatinae Schmiedeknecht, 1910, Phrudinae Townes and Townes, 1949, are newly introduced to Korean fauna. All specimens are based on the insect collection of animal systematic laboratory at the Yeungnam University Gyeongsan Campus. Photographs of habitus of newly recorded subfamilies, diagnosis of 28 species and host information are provided.
\end{abstract}

Keywords: Eucerotinae, Diacritinae, Ichneumoninae, Microleptinae, Orthocentrinae, Orthopelmatinae, Phrudinae, taxonomy

\section{NTRODUCTI ON}

They are small and rare subfamilies of the family Ichneumonidae including. The subfamily Orthocentrinae has been reported to consist of 31 genera with 481 species worldwide, but Korean fauna is poorly known. It includes parts of previously established Microleptinae and Oxytorinae, and all species are known for larval koinobiont endoparasitoids of Diptera (Humala, 2008). The subfamily Diacritinae has been reported to consist of six species worldwide including four Eastern Palaearctic species (Yu et al., 2012). The subfamily Microleptinae monotypic subfamily with 13 species. Among them, 10 species are distributed in the Eastern Palaearctic. The subfamily Orthopelmatinae is also monotypic, and consists of nine species including five Eastern Palaearctic species. The subfamily Phrudinae consists of 29 species, including five species in eastern Palaearctic. The subfamily Eucerotinae contains 50 species worldwide and four species from Korea. This subfamily is known for hyperparasitoids of Ichneumonoidea. The members of this subfamily lay their eggs on leaf surfaces, and their first instar larvae attach to a Lepidoptera or Symphyta larva, and then they enter the body of primary endoparasitoids or attached ectoparasitoids (Wahl and Sharkey, 1993). Only two species of this subfamily are known from Korea (Uchida, 1955, 1958; Lee et al., 1992;
Lee and Cha, 2000). The subfamily Ichneumoninae consists of 4,300 species worldwide, which is the second largest subfamily of the family Ichneumonidae. In Korea, this subfamily was first studied by Uchida (1955), who reported two species of Korean Ichneumoninae. Kim (1970) described 88 species in his illustrated book of Korean Hymenoptera. Yu et al. (2005) listed 97 species in 40 genera of the subfamily Ichneumoninae from Korea, and recently added 21 species (Jeong and Lee, 2006, 2008; Jeong et al., 2007, 2008, 2009, 2010; Jeong and Cha, 2009; Kwon et al., 2012). Including two species reported here in, the Korean Ichneumoninae now comprises 140 species.

In this paper, disital images of habitus of newly recorded subfamilies and diagnoses of newly recorded 28 species from Korea and abailable host information are provided.

\section{MATERI ALS AND METHODS}

Materials used in this study were collected by insect sweeping net and Malaise traps, and they were deposited in the animal systematic laboratory of Yeungnam University (YNU, Gyeongsan, Korea). Specimens were examined by a AxioCam MRc5 camera attached to a stereomicroscope (Zeiss SteREO Discovery. V20; Carl Zeiss, Göttingen, Germany), processed

*To whom correspondence should be addressed

Tel: 82-53-810-2376, Fax: 82-53-811-2376

E-mail: jwlee1@ynu.ac.kr
This is an Open Access article distributed under the terms of the Creative Commons Attribution Non-Commercial License (http://creativecommons.org/ licenses/by-nc/3.0/) which permits unrestricted non-commercial use, distribution, and reproduction in any medium, provided the original work is properly cited.

pISSN 2234-6953 eISSN 2234-8190 
using AxioVision SE64 software (Carl Zeiss), and optimized with a Delta imaging system (i-solution; IMT i-Solution Inc. Vancouver, Canada). The morphological terminology mostly follows that of Townes (1969). Abbreviations are as follows: TD, type depository; TL, type locality; MOMOI, Kobe University, Faculty of Agriculture, Entomological Laboratory, Kobe, Japan. (S. Momoi collection); AEI, American Entomological Institute, 3005 S.W. 56th Avenue, Gainesville, Florida, 32608, USA (H. Townes collection); NM, Naturhistorisches Museum, Stift Admont, A-8911 Admont, Austria (G. Strobl collection); NHM, The Natural History Museum, Department of Entomology, Cromwell Road, London, England, SW7 5BD, United Kingdom; BFIC, Muséum National d'Histoire Naturelle Brunoy, France; BMNH, The Natural History Museum, Department of Entomology, Cromwell Road, London, England; HNHM, Termeszettudomanyi Muzeum Allattara (Hungarian Natural History Museum), Budapest, Hungary; HOPE, Hope Entomological Collection, University Museum, Oxford, England, OX1 3PW, United Kingdom; HU, Entomological Institute, Faculty of Agriculture, Hokkaido University, Sapporo, Japan; IRSNB, Institut Royal des Sciences Naturelles de Belgique, Brussels, Belgium; MZ, Musée Zoologique, Place Riponne, CH-1000 Lausanne, Switzerland; NHM, The Natural History Museum, Department of Entomology, Cromwell Road, London, England, SW7 5BD, United Kingdom; NHRS, Naturhistoriska riksmuseet, Stockholm, Sweden; RIEDEL, Amselweg 9A. Bad Fallingbostel, D-29683, Germany (Matthias Riedel Collection); USNM, U.S. National Museum of Natural History, Smithsonian Institute, Washington, D.C., 20560, U.S.A.; ZSM, Zoologisches Staatsammlung, Münchhausenstrasse 21, D-81247 München, Germany; ZI, Zoologiska Institutionen, Helgonavägen 3, S-223 62 Lund, Sweden; ZIN, Zoological Institute, Academy of Sciences, St. Petersburg 199034, Russia; GW, Gangwon-do; GG, Gyeonggi-do; CB, Chungcheongbuk-do; CN, Chungcheongnam-do; GB, Gyeongsangbuk-do; GN, Gyeongsangnam-do; JB, Jeollabuk-do; JN, Jeollanam-do.

\section{SYSTEMATI C ACCOUNTS}

Order Hymenoptera

Family Ichneumonidae Latreille, 1802

$1 *$ Subfamily Diacritinae Townes, 1965

Diagnosis. Fore wing length $5.0-8.5 \mathrm{~mm}$. Clypeus weakly convex or almost flat, separated from face by transverse carina. Occpital carina complete and very distinguished.
Notaulus long and strong. Propodeum with distinguished pleural carina, lateral area and metapleurum separated by pleural carina. Fore wing with small triangular areolet. Nervellus intercepted below the middle, discoidella present. Petiole very narrow and long. 2nd and 3rd tergites with distinct thyridium.

\section{2* Ortholaba tenuis Townes, 1969 (Fig. 1)}

Ortholaba tenuis Townes, 1969: 129. Type: female; TD: MOMOI.

Material examined. Korea: $1 \sigma^{\nearrow}$, GW: Hyangrobong, 13 Jun 1992, Lee JW [YNU].

Diagnosis. Body length $11.6 \mathrm{~mm}$. Flagella with 28 segments, antennal scape strongly swollen. Central part of face convex. Clypeus small and separated from face by transverse carina, apical margin of clypeus truncated. Notaulus distinct and deep. Hind leg very long and slender, hind coxa longer than propodeum. Fore wing with triangular areolet. Metasoma longer than fore wing.

Host. Unknown.

Distribution. Korea (new record), Japan, Russia.

Remarks. This species is newly included in the Korean fauna. It is easily distinguished by the length of the metasoma three times as long as the mesosoma and 1st tergite straight.

${ }^{3 *}$ Subfamily Eucerotinae Viereck, 1919

Diagnosis. Fore wing length 4.0-11.0 mm. Clypeus not or weakly separated from face, apical margin of clypeus with one obtuse angulation medially. Antennal flagellomeres widened and flattened medially in male. Occpital carina complete and reaching base of mandible. Notaulus strong and short. Tarsal claws pectinate.

4*Euceros schizophrenus Kasparyan, 1984 (Fig. 2)

Euceros schizophrenus Kasparyan, 1984: 78-83. Type: male; TD: ZIN.

Material examined. Korea: 1우, GW: Heungeop-myeon, Maeji-ri, Yeonsedae gyonae ungdeongi yeop, 19 May-6 Jun 2011 [YNU].

Diagnosis. Body length $7.8 \mathrm{~mm}$. Flagella with 31 segments. Central part of face convex. Clypeus wide and flat, weakly separated from face by groove, apical margin of clypeus rounded. Occipital carina very distinct and with deep medial groove. Pronotum mediodorsally with deep v-shape longitudinal furrow. Fore wing without areolet.

Korean name: ${ }^{1 *}$ 긴배마디맵시벌아과 (신칭), ${ }^{2 *}$ 긴배마디맵시벌 (신칭), ${ }^{3 *}$ 넓적수염맵시벌아과, ${ }^{4 *}$ 참넓적수염맵시벌 (신칭) 


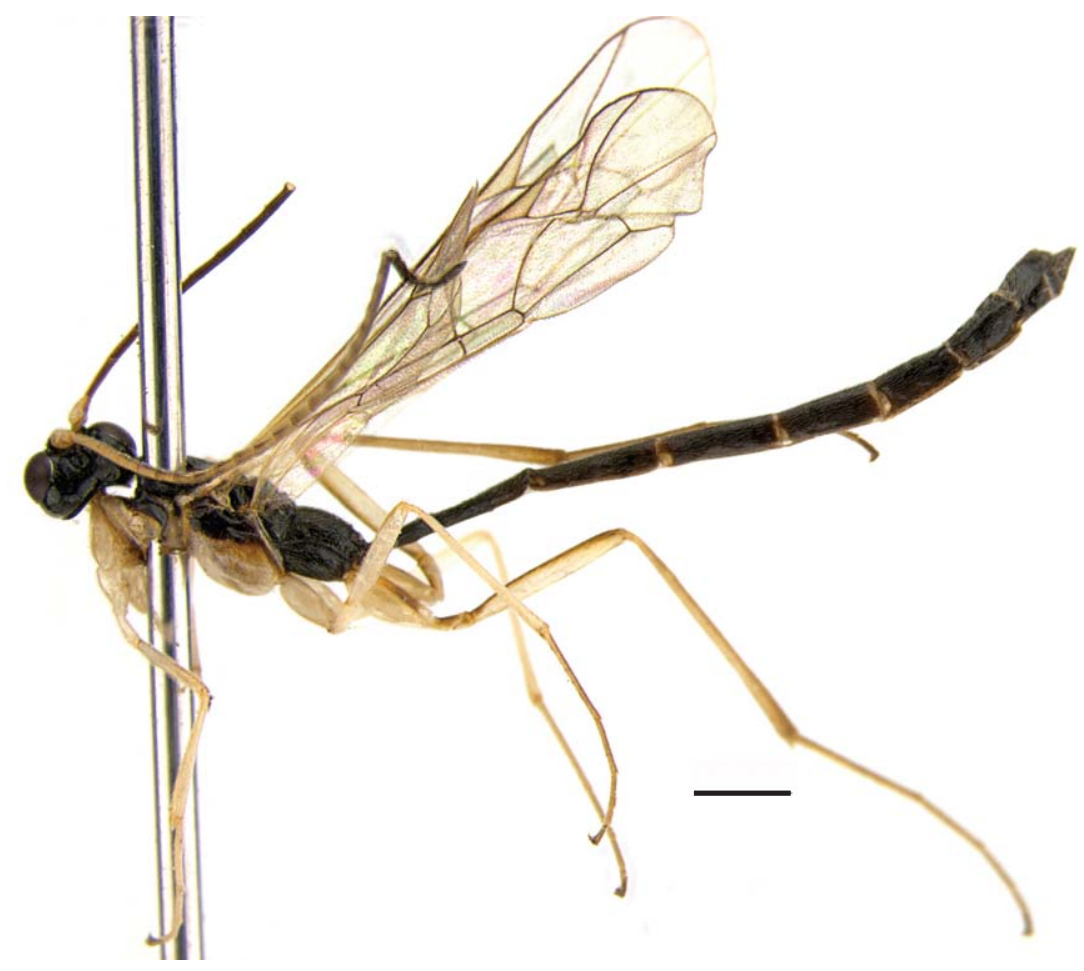

Fig. 1. Habitus of Ortholaba tenuis Townes, 1965 (subfamily Diacritinae). Scale bar=1 mm.

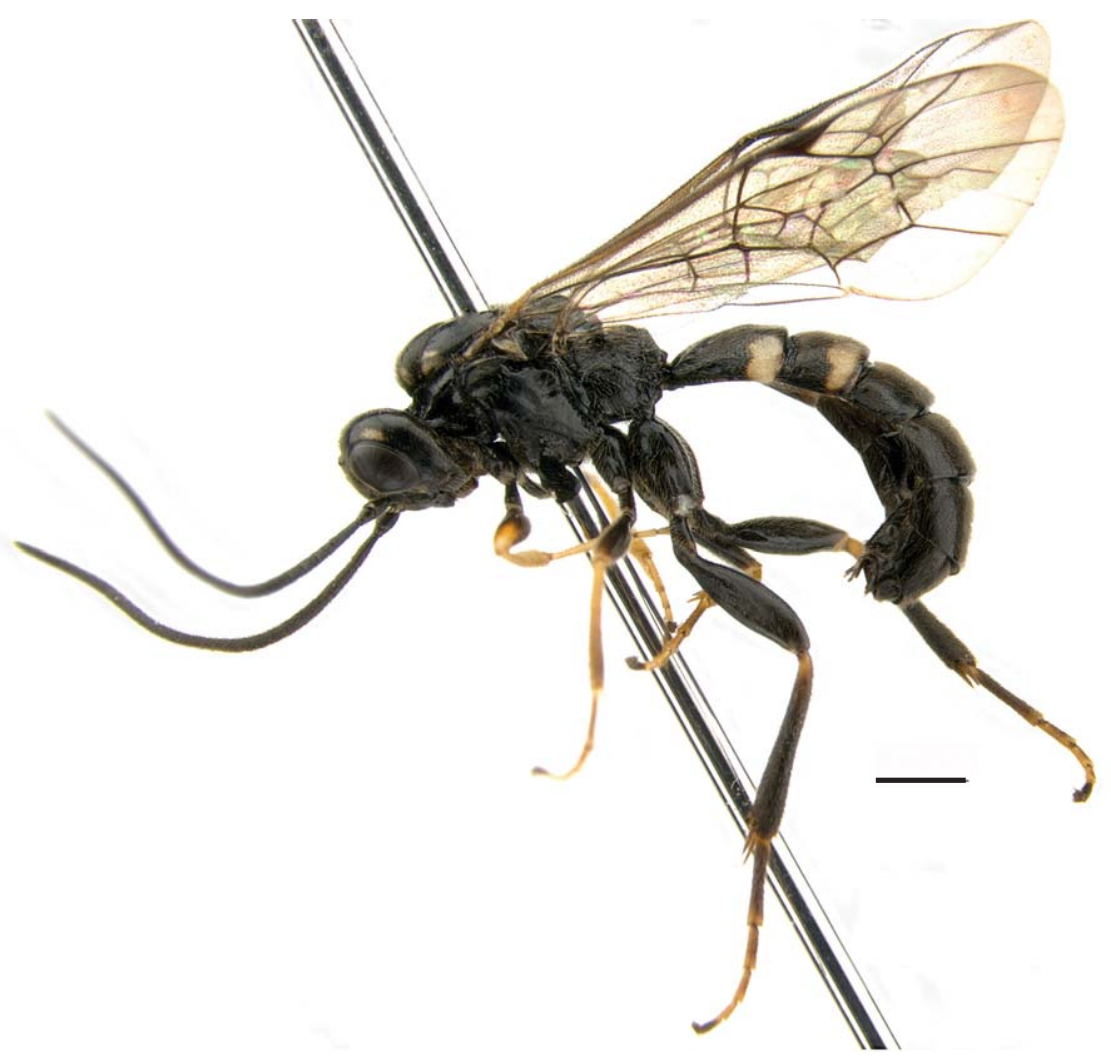

Fig. 2. Habitus of Euceros schizophrenus Kasparyan, 1984 (subfamily Eucerotinae). Scale bar=1 mm. 


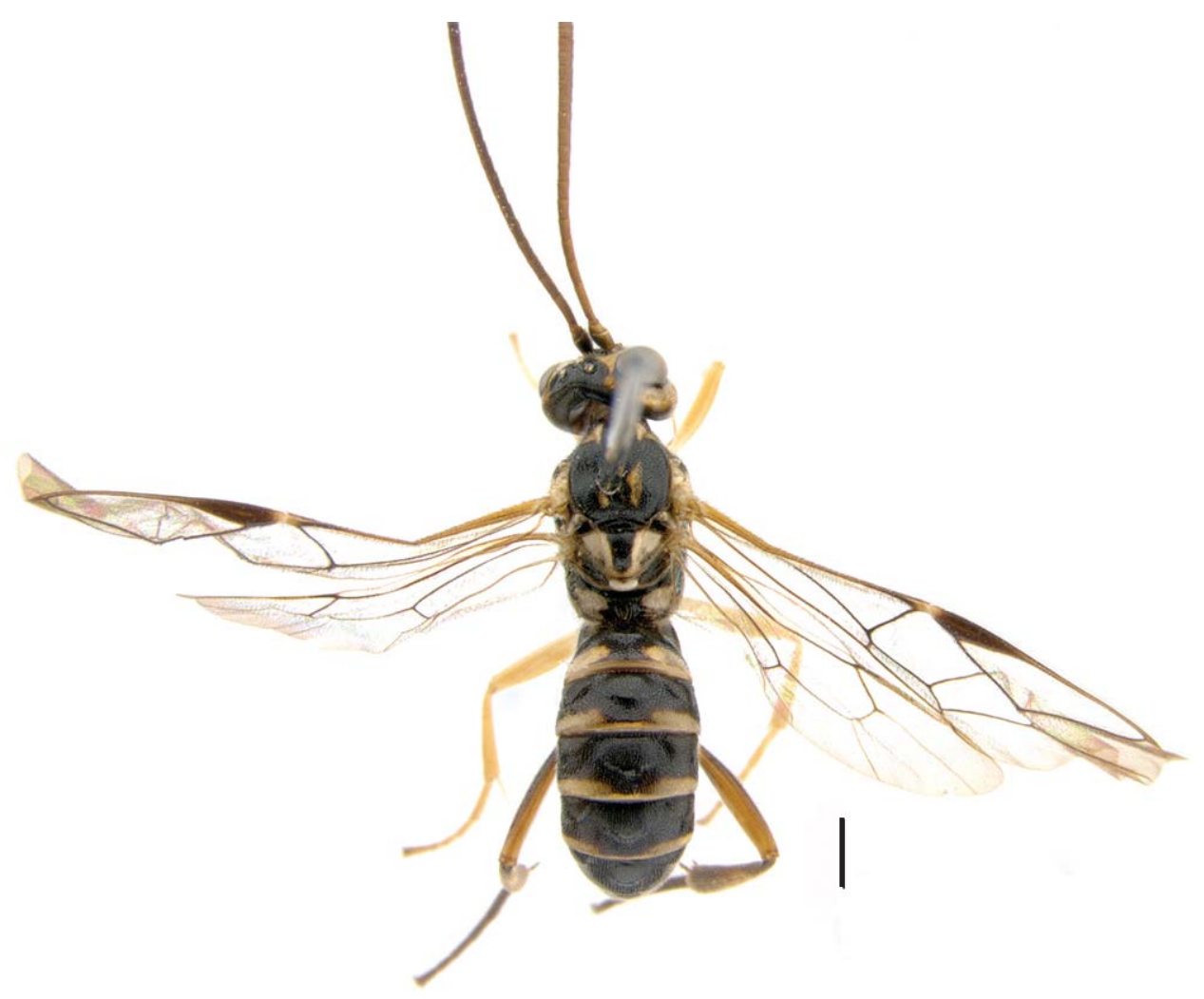

Fig. 3. Habitus of Euceros dentatus Barron, 1978 (subfamily Eucerotinae). Scale bar=1 mm.

Host. Unknown.

Distribution. Korea (new record), Kazakhstan, Russia.

${ }^{1 *}$ Euceros dentatus Barron, 1978 (Fig. 3)

Euceros dentatus Barron, 1978: 327-374. Type: female; TD:

AEI.

Material examined. Korea: 1 우, GG: Pyeongtaek-si, Jangdang-dong, Jangdanggol, $37^{\circ} 2^{\prime} \mathrm{N}, 127^{\circ} 3^{\prime} \mathrm{E}, 11$ Aug-8 Sep 2001, Lee JW [YNU].

Diagnosis. Body length $8.0 \mathrm{~mm}$. Flagella with 40 segments. Central part of face weakly convex. Clypeus small and weakly convex, not separated from face. Occipital carina complete. Pronotum mediodorsally without longitudinal furrow. Propodeum very short. Fore wing without areolet.

Host. Unknown.

Distribution. Korea (new record), China, Russia.

${ }^{2 *}$ Subfamily Microleptinae Townes, 1958

Diagnosis. Fore wing length 4.0-4.8 mm. Clypeus wide and almost flat, separated from face by weak groove. Apex of mandible very wide. Antennal flagellomeres with tyloids in male. Occipital carina complete. Propodeal transverse carina medially incomplete. Fore wing without areolet. Hind tibia with dense setal fringe on posterior apically.

\footnotetext{
3*Microleptes splendidulus Gravenhorst, 1829 (Fig. 4) Microleptes splendidulus Gravenhorst, 1829: 679. Type: unknown; TD: Unknown.
}

Material examined. Korea: $3 \sigma^{\curlyvee} \sigma^{\top}$, GW: Donghae-si, Samhwa-dong, Mureung Valley (M.T.), $37^{\circ} 27^{\prime} \mathrm{N}, 129^{\circ} 1^{\prime} 42.33^{\prime \prime} \mathrm{E}$, 22 Jun-3 Aug 2006, Lee JW [YNU]; $1{ }^{\nearrow}$, Busan: Gijanggun, Jeonggwan-myeon, Dalsan, Gijanggun-cheonsonyeon-

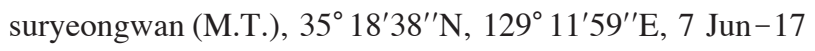
Aug 2008, Lee JW [YNU].

Diagnosis. Body length $3.7 \mathrm{~mm}$. Flagella with 14 segments. Upper part of face convex. Clypeus small and separated from face by weak groove. Apical margin of clypeus rounded.

Host. Chloromyia formosa (Yu et al., 2012).

Distribution. Korea (new record), Bulgaria, Canada, Czechoslovakia, Finland, Germany, Hungary, Ireland, Lithuania,

Korean name: ${ }^{1 *}$ 노랑무늬넓적수염맵시벌 (신칭), ${ }^{2 *}$ 미소맵시벌아과 (신칭), ${ }^{3 *}$ 미소맵시벌 (신칭) 


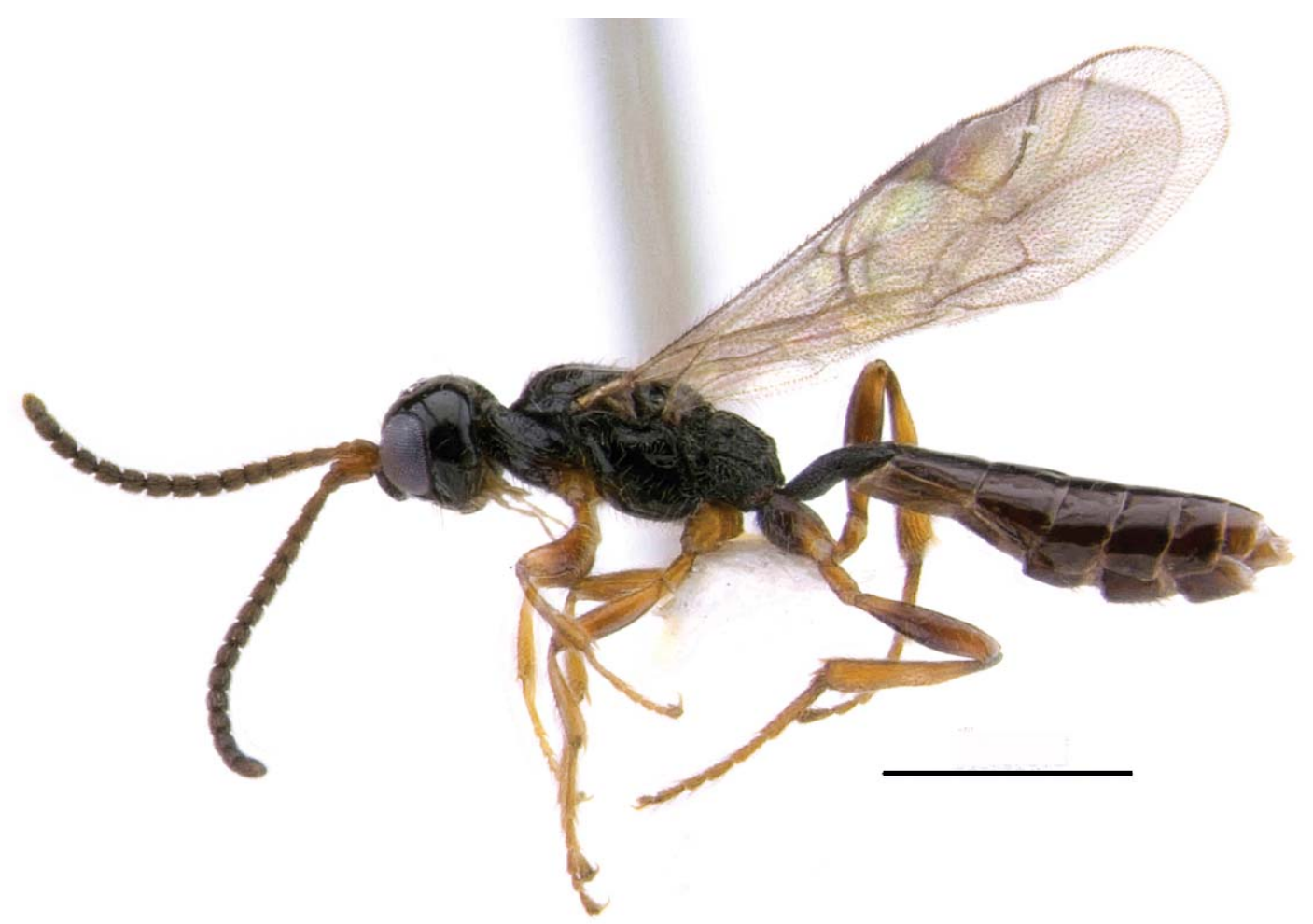

Fig. 4. Habitus of Microleptes splendidulus Gravenhorst, 1958 (subfamily Microleptinae). Scale bar=1 mm.

Netherlands, Norway, Poland, Romania, Russia, Sweden, USA, Ukraine, United Kingdom.

1*Subfamily Orthocentrinae Förster, 1869

Diagnosis. Fore wing length $2.0-9.0 \mathrm{~mm}$. Face very strongly convex. Clypeus strongly convex and small. Mandible slender and thin. Malar space long.

2*Proclitus heterocerus (Thomson, 1888) (Fig. 5)

Plectiscus heterocerus Thomson, 1888: 1307. Type: female; TD: ZI.

Material examined. Korea: 1 우, CN: Buyeo-gun, Gyuammyeon, Sumongni (M.T.), 14-25 May 2005, Lee JW [YNU]. Diagnosis. Body length $3.6 \mathrm{~mm}$. Flagella with 16 segments, slender with hairs. Clypeus small and convex, separated from face by groove. Cubitus of fore wing connected to radius, 1 st intercubitus absent. Ovipositor very long, longer than metasoma, and straight.

Host. Delia radicum (Yu et al., 2012).

Distribution. Korea (new record), France, Germany, Latvia, Poland, Romania, Russia, Sweden.
${ }^{3 *}$ Eusterinx (Ischyracis) bispinosa (Strobl, 1901) (Fig. 6) Hemiteles bispinosus Strobl 1901: 132-257. Type: male; TD: NM.

Material examined. Korea: 1 우, GW: Donghae-si, Samhwa-dong, Mureung Valley (M.T.), 28 Aug-10 Sep 2006, Lee JW [YNU].

Diagnosis. Body length $6.3 \mathrm{~mm}$. Flagella with 18 segments. Clypeus small and convex, separated from by groove. Propodeum with two projections. 1st tergite with longitudinal striae. 2nd tergite and 3rd tergite longitudinally rugose punctated. Ovipositor short.

Host. Orfelia fultoni (Yu et al., 2012).

Distribution. Korea (new record), Austria, Canada, Czecho, Slovakia, Finland, France, Germany, Netherlands, Poland, Russia, Sweden, USA, Ukraine.

${ }^{4 *}$ Subfamily Orthopelmatinae Schmiedeknecht, 1910

Diagnosis. Fore wing length $3.0-4.0 \mathrm{~mm}$. Clypeus weakly convex and small, separated from face by groove, apical margin of clypeus concave. Occpital carina complete. Propodeum with carina. Fore wing without areolet. Nervellus not

Korean name: ${ }^{1 *}$ 긴뺨맵시벌아과 (신칭), ${ }^{2 *}$ 긴 꼬리긴뺨맵시벌 (신칭), ${ }^{3 *}$ 쌍가시긴뺨맵시벌 (신칭), ${ }^{4 *}$ 원통배자루맵시벌아과 (신칭) 


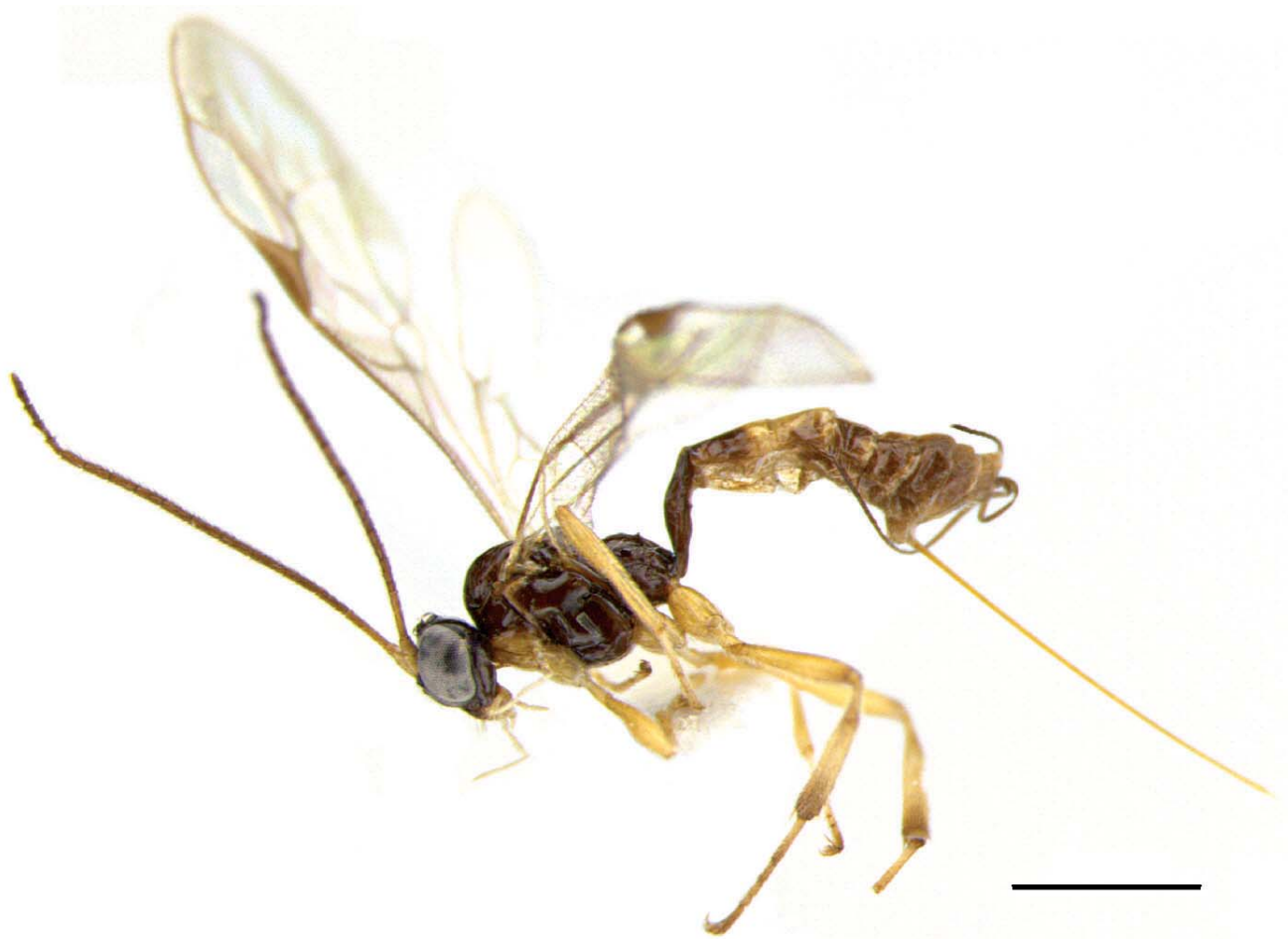

Fig. 5. Habitus of Proclitus heterocerus (Thomson, 1888) (subfamily Orthocentrinae). Scale bar=1 mm.

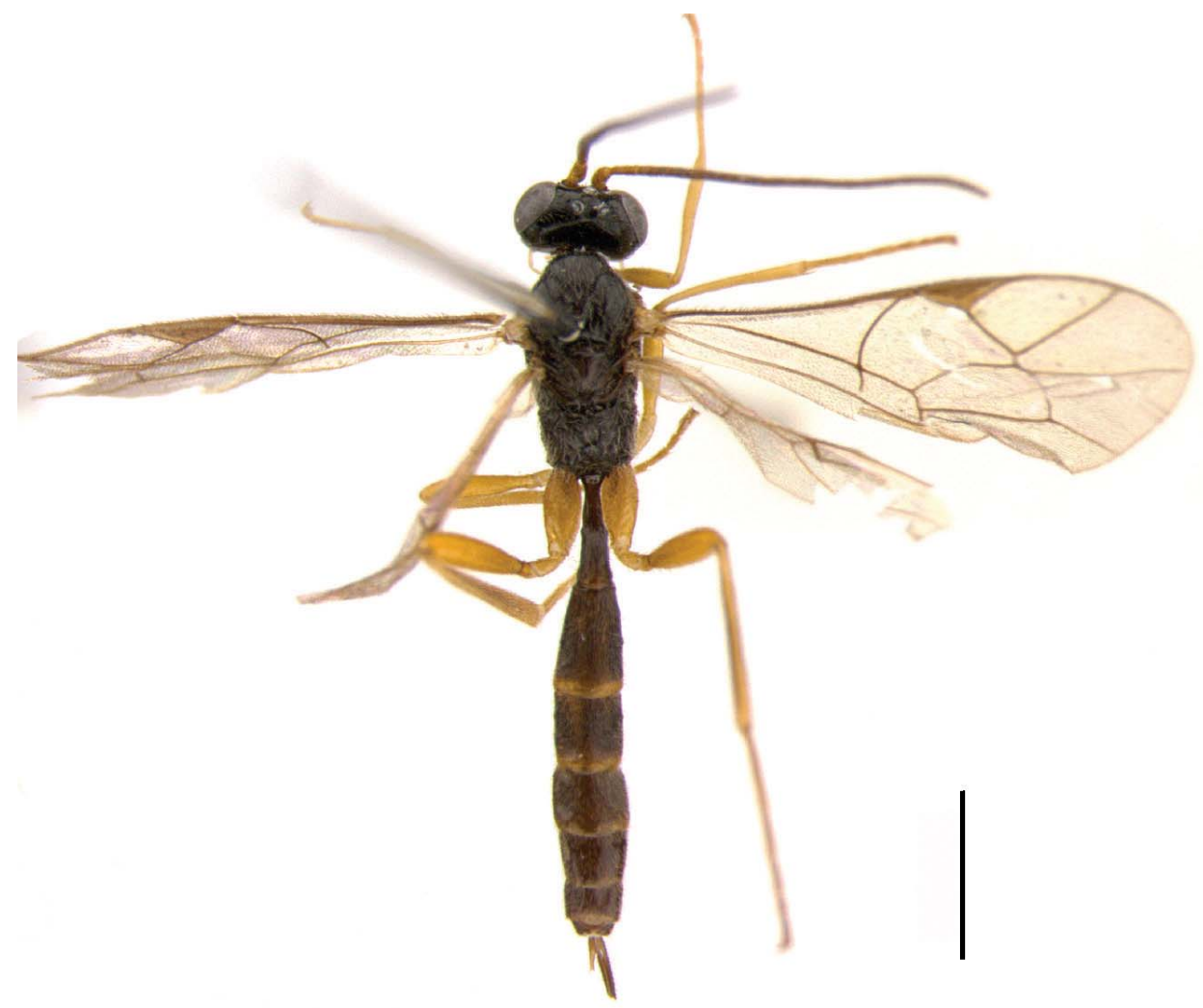

Fig. 6. Habitus of Eusterinx (Ischyracis) bispinosa (Strobl, 1901) (subfamily Orthocentrinae). Scale bar=1 mm. 


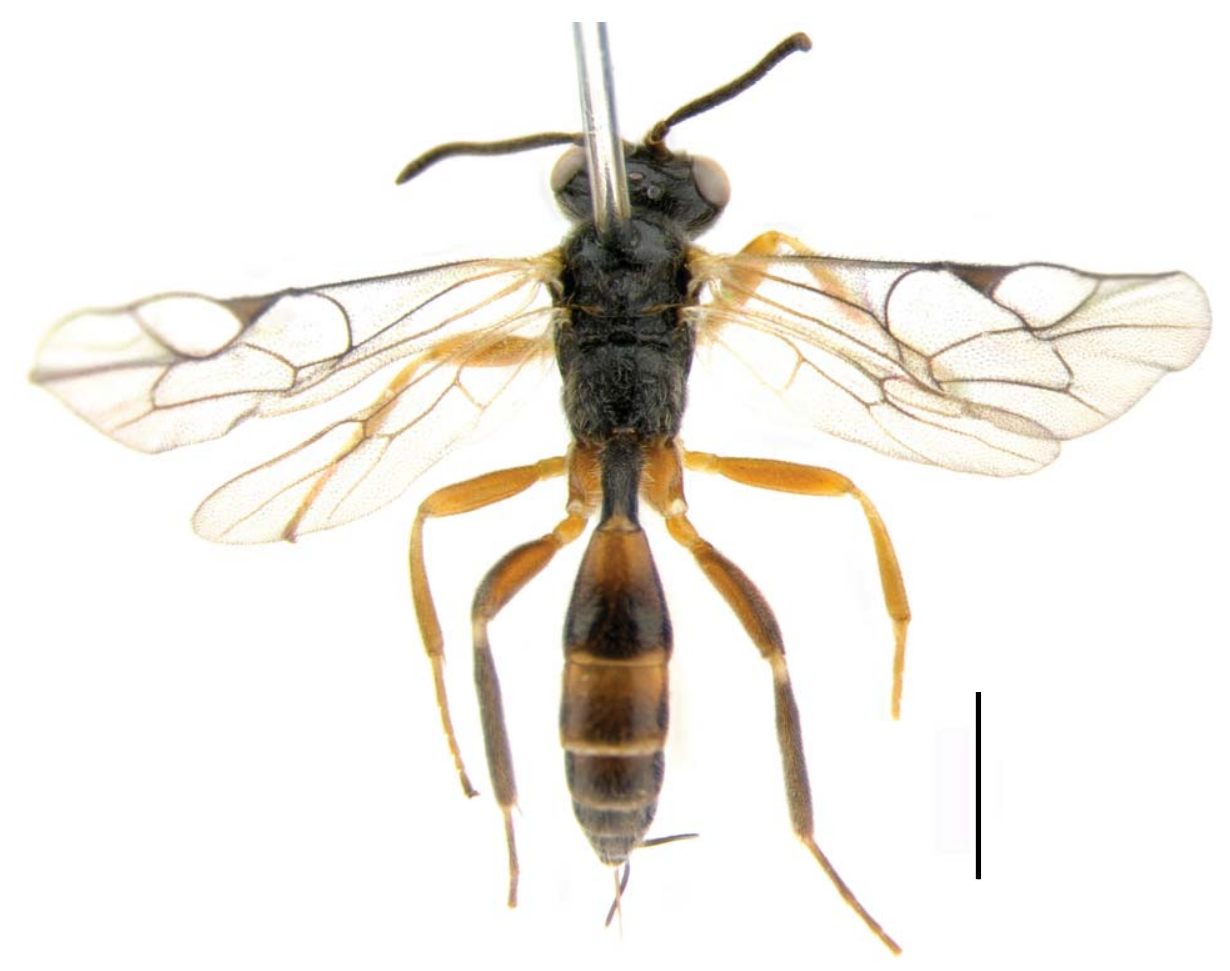

Fig. 7. Habitus of Orthopelma brevicorne Morley, 1907 (subfamily Orthopelmatinae). Scale bar=1 mm.

intercepted, discoidella absent. Petiole cylindrical and 1st tergite as long as 1 st sternite.

1*Orthopelma brevicorne Morley, 1907 (Fig. 7)

Orthopelma brevicorne Morley, 1907: 112. Type: female; TD: NHM.

Material examined. Korea: 1 우, GW: Chuncheon-si, Sanong-dong, Gwangwon Prov., Arboretum, alt. $81 \mathrm{~m}, 37^{\circ} 55^{\prime}$ 23. $7^{\prime \prime} \mathrm{N}, 127^{\circ} 43^{\prime} 7^{\prime \prime} \mathrm{E}, 2-15$ May 2012, Lee GY [YNU]; 2 우 우, Daejeon: Youngun-dong, Daejeon Univ., 12-27 May 2007, Lee JW [YNU]; 1 우, Daegu: Dalseo-gu, Daegok-dong, Daegu Arboretum, alt. 88 m, $37^{\circ} 47^{\prime} 48.6^{\prime \prime} \mathrm{N}, 128^{\circ} 31^{\prime} 33.5^{\prime \prime} \mathrm{E}$, 3-16 May 2012, Kang SG [YNU].

Diagnosis. Body length $3.9 \mathrm{~mm}$. Flagella with 15 segments, antennal pedicel weakly swollen. Central part of face convex. Clypeus small and convex, separated from face by transverse carina. 1st tergite with spiracle on basal one-third. 1st tergite with scattered punctures on basal three-fifths part and longitudinal striae on remaining part.

Host. Diplolepis dispar, Diplolepis eglanteriae, Diplolepis rosae, Diplolepis spinosissimae (Yu et al., 2012).

Distribution. Korea (new record), Bulgaria, Finland, France, Russia, Spain, Sweden, Turkey, United Kingdom.
${ }^{2 *}$ Subfamily Phrudinae Townes \& Townes, 1949

Diagnosis. Fore wing length 2.0-26.0 mm. Clypeus large, separated from face by groove, apical margin of clypeus thickeded. Propodeum with carina, areola usually complete but in some species almost absent. Fore wing with areolet, stigma large and triangular. Lateral tergites of 3rd (sometimes also 2 nd) to 6 th tergites not separated by crease.

${ }^{3 *}$ Phrudus badensis Hilpert, 1987 (Fig. 8)

Phrudus badensis Hilpert, 1987: 213-219. Type: female; TD: ZSM.

Material examined. Korea: 1 우, GW: Heungeop-myeon, Maeji-ri, Yeonsei Univ., Jeorgsang, $37^{\circ} 16^{\prime} 53^{\prime \prime} \mathrm{N}, 127^{\circ} 54^{\prime}$ 02'E, 16 May-6 Jun 2011 [YNU]; 1 우, Daegu: Dalseo-gu, Daegok-dong, Daegu Arboretum, alt. $88 \mathrm{~m}, 37^{\circ} 47^{\prime} 48.6^{\prime \prime} \mathrm{N}$, $128^{\circ} 31^{\prime} 33.5^{\prime \prime} \mathrm{E}, 16-30$ May 2012, Kang SG [YNU]; 1 우, ditto, 30 May-7 Jun 2012, Kang SG [YNU].

Diagnosis. Body length $3.1 \mathrm{~mm}$. Flagella with 13 segments. Face with fine transverse striae. Clypeus wide, separated from face by transverse groove. Lateral part of 1st tergite with longitudinal striae; remaining terga glabrous.

Host. Unknown.

Korean name: ${ }^{1 *}$ 짧은원통배자루맵시벌 (신칭), ${ }^{2 *}$ 애맵시벌아과 (신칭), ${ }^{3 *}$ 애맵시벌 (신칭) 


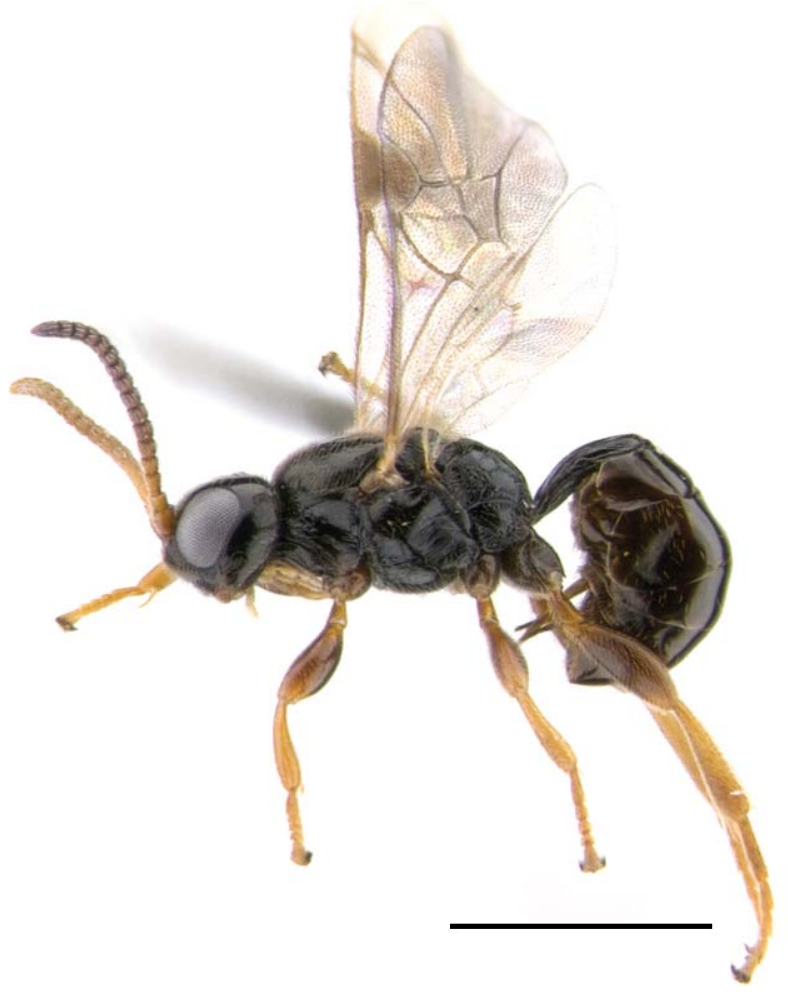

Fig. 8. Habitus of Phrudus badensis Hilpert, 1987 (subfamily Phrudinae). Scale bar $=1 \mathrm{~mm}$.

Distribution. Korea (new record), Finland, Germany, Italy, Poland, Russia, United Kingdom.

${ }^{1 *}$ Subfamily Ichneumoninae Latreille, 1802

Diagnosis. Fore wing length 2.2-21.0 mm. Clypeus wide and flat, separated from supra-clypeal area by weak groove. Fore wing with almost closed pentagonal or subtriangular areolet. 1st tergite slender anteriorly and widened posteriorly. 2nd tergite usually with distinct and deep gastrocoelus.

\section{2*Asthenolabus semimarginalis Uchida, 1930}

Platylabus semimarginalis Uchida, 1930: 98. Type: female;

TL: Minomo, Japan; TD: HU.

Platylabus semimarginalis: Meyer, 1933: 376.

Asthenolabus semimarginalis: Townes, Momoi \& Townes, 1965: 514; Yu \& Horstmann, 1997: 674.

Material examined. Korea: 1 우, GN: Mt. Jirisan, Cheonghak, Samsanbong, Jinjuam, 8 Jul-1 Sep 2001, Lee JW [YNU]; 1 우, Mt. Jirisan, Jangdanggol, Anjangdang, 11 Aug-8 Oct 2001, Lee JW [YNU].

Diagnosis. Body length $10 \mathrm{~mm}$. Fore wing length $8 \mathrm{~mm}$.
Flagella with 37 segments, lanceolate from 13 segment, central flagellomeres of female elongate. Clypeus swollen, small and subquadrate, uniformly thick. Clypeus with punctures evenly distributed, apical margin evenly round, without a median apical tubercle but weakly angled. Scutellum strongly convex, shelf-like; lateral longitudinal carinae more or less complete to center or beyond, but not reaching posterior margin. 1st metasomal segment in lateral view with flattened petiole basally and convex postpetiole; basal area of petiole without projections. Gastrocoeli 0.8 times as broad as distance between them, and with distinctly impressed thyridium.

Host. Unknown.

Distribution. Korea (new record), Japan.

3*Asthenolabus wadai (Uchida, 1935)

Platylabus wadai Uchida, 1935: 33. Type: female; TL: Hongawa, Japan; TD: HU.

Platylabus wadai: Townes, Momoi \& Townes, 1965: 514; Yu \& Horstmann, 1997: 674.

Material examined. Korea: 1 우, GW: Inje-gun, Mt. Bangdaesan, Misan-ri, 25 Jun 1996, Lee JW [YNU]; 1 우, Donghae-si, Samhwa-dong, Muryeung Valley, 4-15 Jul 2005,

Korean name: ${ }^{1 *}$ 맵시벌아과, ${ }^{2 *}$ 검정납작배자루진맵시벌 (신칭), ${ }^{3 *}$ 와다납작배자루진맵시벌 (신칭) 
Lee JW [YNU].

Diagnosis. Body length $10 \mathrm{~mm}$. Fore wing length $8 \mathrm{~mm}$. Flagella with 38 segments, weakly lanceolate from 15 segment, central flagellomeres of female elongate. Clypeus basally slightly swollen, slightly concave apically, small and subquadrate, uniformly thick. Clypeus with punctures evenly distributed, apical margin simple, without median apical tubercle. Scutellum strongly convex, shelf-like; lateral longitudinal carinae more or less complete to center or beyond, but not reaching posterior margin. Gastrocoeli 0.8 times as broad as distance between them, and with distinctly impressed thyridium.

Host. Unknown.

Distribution. Korea (new record), Japan.

${ }^{1 *}$ Auberteterus alternecoloratus (Cushman, 1929)

Centeterus alternecoloratus Cushman 1929: 243. Type: female; TL: Fujian, China; TD: USNM.

Centeterus rufocyanator Aubert, 1964: 147. Type: male; TL: France-main; TD: MZ.

Material examined. Korea: 1 우, GB: Gyeongju-si, Wetland of Tohamsan, 27 Aug 2008, Jeong JC [YNU].

Diagnosis. Apical edge of clypeus straight, above two blunt teeth. Mandibles and temples very broad. Vein 3rm present. Propodeum gradually sloping from base to apex. Area superomedia long and slender. Thyridiae placed at base of tergite 2 . Host. Chilo auricilius, Chilo infuscatellus, Chilo suppressalis, Chilo zonellus (Yu et al., 2012).

Distribution. Korea (new record), China, France, India, Russia.

\footnotetext{
$2 *$ Barichneumon arakawai (Uchida, 1925)

Aglaojoppa formosana ab. arakawai Uchida, 1925a: 245.

Type: male; TL: Hachijo Island, Japan; TD: HU.

Aglaojoppa formosana var. arakawai: Uchida, 1926: 61.

Melanichenumon arakawai: Uchida, 1932: 138.

Barichneumon arakawai: Townes, Townes \& Gupta, 1961:

363; Townes, Momoi \& Townes, 1965: 434; Gupta, 1987:

988; Takahashi, 1995: 7; Yu \& Horstmann, 1997: 542.
}

Material examined. Korea: 1 ð , Daegu: Mt. Apsan, 27 May 1990, Quan YJ [YNU]; 1 ð , CN: Buyeo-gun, Sumok-ri, 1-5 Jun 2005, Lee JW [YNU].

Diagnosis. Body length $14 \mathrm{~mm}$. Fore wing length $10 \mathrm{~mm}$. Flagella with 38 segments, with tyloids at segments 7-20, central flagellomeres quadrate. Clypeus flat, in same plane as lower face, simply transverse, uniformly thick. Scutellum moderately convex; lateral longitudinal carinae more or less complete to center. Gastrocoeli 0.6 times as broad as distance between them, and with distinctly impressed thyridium.

Host. Unknown.

Distribution. Korea (new record), Japan, Taiwan.

${ }^{3 *}$ Barichneumon tosaensis (Uchida, 1935)

Ichneumon vexator Smith, 1874: 390. Type: female; TL: Japan; TD: BMNH. Homonym of Ichneumon vexator Thunberg, 1824.

Ichneumon vexator: Uchida, 1926: 84.

Melanichneumon leucomelas f. tosaensis Uchida, 1935: 18. Type: female; TL: Fukui, Japan; TD: HU.

Melanichneumon vexator: Heinrich, 1937: 269; Uchida, 1939:

34; 1953: 127; Iwata, 1958: 71; 1960a: 41; 1960b: 136.

Barichneumon vexator: Townes, Momoi \& Townes, 1965: 438; Kim \& Lee, 1982: 52.

Barichneumon leucomelas: Kim, 1970: 390.

Barichneumon tosaensis: Yu \& Horstmann, 1997: 546.

Material examined. Korea: 1 우, GG: Ongjin-gun, Deokjeokdo, Seopo-ri, 6 Jul 1981, Lee KS [YNU]; 1 우, Paju-gun, Daeseong-dong, 30 Jun 1987, Yu HJ [YNU]; 1 우, Gwacheonsi, Mt. Cheonggyesan, 30 May 1991, Lee JR [YNU]; 1 우, GW: Hwacheon-gun, 6 Jun 1967, Oh JK [YNU]; 1 우, GN: Miryang-si, Pyochungsa, Sajapyeong, 14-20 May 2003, Jeong JC [YNU]; $1 \sigma^{\top}$, JN: Buyeo-gun, Guam-myeon, Sumokri, 1-15 Jun 2005, Lee JW [YNU]; 1 우, Mt. Jirisan, Sunduryu, 3 Jun 1989, Baek JS [YNU]; 1 우, ditto, Park JS [YNU]; 1 우, ditto, Cha JY [YNU]; 1 우, ditto, Lee JW [YNU].

Diagnosis. Body length $16 \mathrm{~mm}$. Flagella with 39 segments. Ground body color black, but median band of flagella, supraantennal orbit, lateral area of clypeus, scutellum, and apical marks of tergites 6-7 yellow. 3rd flagellomere 2.0 times as long as its width. Scutellum flat, without lateral carina. Areola hexagonal, well defined by carinae. Median field of postpetiole closely punctate.

Host. [Lepidoptera] Arctiidae: Hyphantria cunea (Drury). (Yu et al., 2012).

Distribution. Korea (new record), Japan, China.

\section{4*Coelichneumon (Coelichneumon) octoguttatus Uchida, 1925}

Coelichneumon 8-guttatus Uchida, 1925b: 450. Type: female;

TL: Sapporo, Japan; TD: HU.

Coelichneumon 8-guttatus: Uchida, 1926: 57; 1936: 72.

Ichneumon octoguttatus: Townes, Momoi \& Townes, 1965: 528.

Coelichneumon (Coelichneumon) octoguttatus: Yu and Horstmann, 1997: 520.

Korean name: ${ }^{1 *}$ 두이빨머리방패진맵시벌 (신칭), ${ }^{2 *}$ 아라카와진맵시벌 (신칭), ${ }^{3 *}$ 두점머리방패 혹진맵시벌 (신칭), ${ }^{4 *}$ 작은팔점진맵시벌 (신칭) 
Material examined. Korea: 1 우, Seoul: Mt. Dobongsan, 25 Aug 1982, Kim HK [YNU]; $10^{7}$, Korea Univ., Imeopsiheomjang, 29 May 1987, Lee JW [YNU]; 1우, GW: Inje-gun, Misan-ri, Mt. Bangtaesan, 25 Jun 1996, Lee JW [YNU]; 1 우, Wonju-si, Heungeop-myeon, Maeji-ri, Yeonse Univ., 10 May 2005, Song YJ, Do JH [YNU]; 1 우, Wonju-si, Heungeop-myeon, Maeji-ri, Mt. Dukgasan, 28 Jul 1998, Byun HW, Choi DS, Kim SK [YNU]; 1우, CB: Mt. Uamsan, 28 May 2002, Shin DS, Baeg SH [YNU]; 1 우, Yeongdong, 31 May 1992, Han SY [YNU]; 1 o $^{7}$, JB: Mt. Naejangsan, 4 Jul 1988, Choi SC [YNU]; Japan: 1 우, Hokkaido, Sapporo, Maruyama, 15 Oct 1922, Uchida T (lectotype of Coelichneumon octoguttatus Uchida, 1925) [HU].

Diagnosis. Body length $18 \mathrm{~mm}$. Fore wing length $13 \mathrm{~mm}$. Central part of face convex. Clypeus flat, with weak median apical tubercle and weakly angled. Scutellum weakly convex; lateral longitudinal carinae absent. Center of outer part of tibia with yellow spot. 1st tergite with longitudinal striae, 2nd tergite with very distinctly impressed thyridium. Metasoma with eight yellow spots.

Host. Unknown.

Distribution. Korea (new record), Japan.

${ }^{1 *}$ Coelichneumon eburnifrons (Wesmael, 1857)

Ichneumon eburnifrons Wesmael, 1857: 355-426. Type: unknown; TL: Belgium; TD: Unknown.

Ichneumon puerulus Kriechbaumer 1890: 181-185. Type: female; TL: Germany; TD: ZSM.

Ichneumon tenuitarsis Thomson 1893: 1889-1967. Type: female; TD: ZI.

Coelichneumon pictus Roman 1904: 115-120. Type: female; TL: Sweden; TD: Unknown.

Ichneumon zagoriensis Schmiedeknecht 1928: 1-272. Type: female; TL: Yugoslavia; TD: Unknown.

Coelichneumon pumilionobilis Heinrich 1951: 235-290. Type: female; TL: Germany; TD: ZSM.

Material examined. Korea: $1 \sigma^{\Im}$, GN: Mt. Jirisan, Georim, Seseoksanjang area, Malaise trap, 20 Jul-12 Oct 2001, Lee JW [YNU].

Diagnosis. Body length $13 \mathrm{~mm}$. Flagellum with 33 segments, small and long-oval tyloids on segments 7-17. Temple strongly narrowed behind eye. Scutellum as long as wide, with lateral carina on the basal half. Lateral field of postpetiole about 0.5 times as wide as the median one, median field with fine striae. Thyridia somewhat oblique, very large, as wide as their interval.

Host. Plagodis pulveraria (Yu et al., 2012).

Distribution. Korea (new record), Austria, Belarus, Belgium,
Canada, Croatia, Czech Republic, Czecho, Slovakia, Finland, France, Germany, Hungary, Norway, Poland, Romania, Russia, Sweden, USA, United Kingdom, Yugoslavia.

${ }^{2 *}$ Coelichneumon opulentus (Taschenberg, 1871)

Ichneumon opulentus Taschenberg, 1871: 307. Type: female; TL: Austria; TD: Unknown.

Ichneumon flaviger Brischke, 1878: 35-117. Type: female. Ichneumon gerstaeckeri Kriechbaumer, 1889: 142-144. Type: male; TL: Austria; TD: ZSM.

Coelichneumon incertatae Uchida, 1956: 58. Type: male; TL: Japan; TD: HU.

Material examined. Korea: $2 \sigma^{\pi} \sigma^{7}$, GG: Namyangju-si, Choan, Songchon, Mt. Ungilsan, alt. 99 m, 27 May-10 Jun 2009, Lim JO [YNU]; $10^{7}$, Yangpyeong-gun, Yongmun, Yeonsu, Mt. Yongmunsan, alt. 324 m, 26 Jun-16 Jul 2009, Lim JO [YNU].

Diagnosis. Juncture of hypostomal and occipital carinae separated from mandibular base. Scutellum yellow, flat, weakly raised above postscutellum or same height; lateral longitudinal carinae of scutellum absent. Propodeum evenly convex. Mesopleura and propodeum with yellowish marks. Areola within general convex surface of propodeum, evenly raised. Coxa, femora, and tibia with yellowish area, tibia yellow with brown apex. Metasoma completely bluish-black. Host. Acronicta intermedia, Panthea coenobita ( $\mathrm{Yu}$ et al., 2012).

Distribution. Korea (new record), Austria, Belarus, Czech Republic, Czechoslovakia, Finland, Germany, Japan, Latvia, Lithuania, Norway, Poland, Russia, Sweden.

\section{${ }^{3 *}$ Cratichneumon aspratilis Townes, Momoi \& Townes, 1965}

Cratichneumon aspratilis Townes, Momoi, Townes 1965: 441. Type: unknown.

Togea nigra Uchida 1926: 112. Type: female; TL: Japan; TD: HU.

Material examined. Korea: 1우, GW: Mt. Gyebangsan, 14 Jul 1995, Park HC [YNU]; 1?, GB: Cheongdo-gun, Unmunmyeon, Mt. Unmunsan, 1-24 Jul 2008, Lee JW [YNU]; $10^{-1}$, JN: Jeongeup-si, Jangseong-gun, Bukha-myeon, Sajabong, 21 Jun 2005, Lee JW [YNU].

Diagnosis. Body length $13 \mathrm{~mm}$. Fore wing length $10 \mathrm{~mm}$. Flagella with 39 segments. Central part of face convex. Clypeus flat, without median apical tubercle. Scutellum weakly convex; lateral longitudinal carinae absent. Propodeum with two projections. 2nd tergite with distinctly impressed thyridium.

Korean name: ${ }^{1 *}$ 세석진맵시벌 (신칭), ${ }^{2 *}$ 가슴무늬보라진맵시벌 (신칭), ${ }^{3 *}$ 뾰족혹뿌리진맵시벌 (신칭) 
Host. Peribatodes secundaria (Yu et al., 2012).

Distribution. Korea (new record), Japan.

\section{${ }^{1 *}$ Cratichneumon insolitus (Walker, 1874)}

Ichneumon insolitus Walker, 1874: 301-310. Type: female; TL: Japan; TD: NHM.

Melanichneumon femoratus Uchida, 1926: 104. Type: female; TL: Japan; TD: HU.

Cratichneumon teranishii Uchida, 1935: 25. Type: female; TL: Japan; TD: HU.

Material examined. Korea: 1 우, GW: Donghae-si, Samhwa-dong, Mureung Valley, 31 Jul-10 Sep 2005, Lee JW [YNU].

Diagnosis. Body black. Flagella with 25 segments, with white band. Mandible red brown. Inner margin of compound eye yellow. Fore tibia white, fore tarsus brown, basal half of mid and hind tibia white. Apical margin of each tergites red brown. Mesosoma and 1st to 2nd tergites coarsely punctate. Clypeus small, weakly round apically. Mesonotum flat, notauli short and weak. Metasoma strongly pointed apically. Gastrocoelus small and weak. Postpetiole with coarse punctures.

Host. Unknown.

Distribution. Korea (new record), China, Japan.

\author{
${ }^{2 *}$ Darachosia rufipes (Cameron, 1903) \\ Enchisiades rufipes Cameron 1903: 220. Type: female; TL: \\ India; TD: HOPE. \\ Enchisiades rufipes: Heinrich, 1937: 263.
}

Material examined. Korea: $2 \sigma^{\nearrow} \sigma^{\nearrow}$ CB: Mt. Sobaeksan, Danyang-gun, Cheondong-ri (Malaise trap), 28 Jul-13 Aug 2006, Lee JW [YNU].

Diagnosis. Body length $15-17 \mathrm{~mm}$. Flagella with 32 segments, slender, all segments much longer than wide, first flagellomere about 3.8-4.0 times as long as wide. Tyloids on segments 11-20, long oval, situated centrally. Face and clypeus with superficial puncture, clypeus flat, with sharp apical margin, apex concave in middle. Scutellum moderately elevated, longer than wide, with complete lateral carina. Area petiolaris with parallel lateral carinae and transverse rugae. Areolet almost quadrangular. Gastrocoeli distintcly impressed, thyridiae oblique.

Host. Unknown.

Distribution. Korea (new record), China, India.

\section{3*Eupalamus japonicus Uchida, 1926}

Eupalamus tretepohlii var. japonicus Uchida, 1926: 115. Lectotype: female; TL: Hokkaido, Japan; TD: HU.
Eupalamus lamentator var. japonicus: Uchida, 1927: 203.

Eupalamus japonicus: Iwata, 1958: 69; 1960b: 134; Townes, Momoi \& Townes, 1965: 448; Yu \& Horstmann, 1997: 577 .

Material examined. Korea: 1 우, Seoul: Gangnam-gu, Mt. Daemosan, 19 Aug 1996, Kim JE [YNU]; $1 \sigma^{7}$, GG: Mt. Myeongjisan, Gwimokgogae-Imsan, 29 Jun 1999, Ryu SM [YNU]; 1 ऽ , Ganghwa, Jeongreungsa, 12 Aug 1991 [YNU]; 1 우, GW: Donghae-si, Samhwa-dong, Mureung Valley, 31 Jul-10 Sep 2005, Lee JW [YNU]; $1 \sigma^{\nearrow}$, ditto, 16-28 Jun 2005, Lee JW [YNU]; 1 ð , GW: Mt. Taebaeksan, 14 May 1992, Lee SJ [YNU]; $1 \sigma^{\nearrow}$, Daejeon: Secheon-gun, 6 Jun 1989, An IS [YNU]; 2 ఠ ఠ , CB: Dannyang-gun, Cheondongri, Mt. Sobaeksan, Bukbu office, 8 Jun-6 Jul 2005, Lee JW [YNU]; $1 \sigma^{\nearrow}$, Mt. Sobaeksan, Birobong, 30 Jul 1988, Kim HG [YNU]; 1 ๙ , CN: Buyeo-gun, Guam-myeon, Sumok-ri, 1-15 Jun 2005, Lee JW [YNU]; 3 ఠ శ , ditto, 27 Jun-12 Jul 2005 [YNU]; 1 우, GB: Gimcheon-si, Jikjisa, 5 Jun 1968, Park EG [YNU]; 1 ð , Pohang-si, Jukjang-myeon, Haok, 23 Jun 2004, Baek MG [YNU]; 1 ð, GN: Mt. Jirisan, Jangdanggol, 12 Jun-11 Jul 2001, Lee JW [YNU]; $2 \sigma^{\nearrow} \diamond^{\nearrow}$, Mt. Jirisan, Jangdanggol, Baggatjangdang, 11 Jul-8 Nov 2001, Lee JW [YNU]; 1 우, JB: Jeongeup-si, Naejang-dong, Mt. Naejangsan, Geumseong, 29 Jul 2005, Lee JW [YNU]; $1 \sigma^{\nearrow}$, Jeongeup-si, Ibam maepyoso, 21 Aug 2004, Han JG [YNU]; 1 જ , Jeongeup-si, Ssangam-dong, Dapgok, 18 Jun 2004, Yun MK [YNU].

Diagnosis. Body length $18 \mathrm{~mm}$. Fore wing length $13 \mathrm{~mm}$. Flagella with 41 segments, lanceolate. Clypeus flat, in same plane as lower face, simply transverse, uniformly thick. Scutellum flat; lateral longitudinal carinae absent or only discernible on extreme anterior end. Gastrocoeli half as broad as distance between them, and with not distinctly impressed thyridium.

Host. Unknown.

Distribution. Korea (new record), Japan.

4*Exephanes apicalis Uchida, 1926

Exephanes apicalis Uchida 1926: 74. Type: female; TL: Japan; TD: HU.

Material examined. Korea: 1 우, CB: Mt. Wolaksan, office of Wolaksan Natural Park, 22 Apr-6 May 2006, Jeong JC [YNU].

Diagnosis. Body length $12 \mathrm{~mm}$. Fore wing length $9 \mathrm{~mm}$. Flagella with 41 segments, bristle shaped, with 3rd flagellomere about twice as long as wide, central flagellomeres quadrate. Flagella with median white band, ventral area of

Korean name: ${ }^{1 *}$ 꼬마혹뿌리진맵시벌 (신칭), ${ }^{2 *}$ 천동진맵시벌 (신칭), ${ }^{3 *}$ 일본닮은혹뿌리진맵시벌 (신칭), ${ }^{4 *}$ 긴꼬리끝무늬진맵시벌 (신칭) 
apical segments brown. Clypeus about twice as wide as long; punctures evenly distributed; apical margin simple. Areola large, quadrate, well-defined by carinae. Postpetiole with distinct median field, with longitudinal striate. Gastrocoeli 0.25 times as broad as distance between them, and with without thyridium.

Host. Chortodes elymi, Mesoligia furuncula (Yu et al., 2012). Distribution. Korea (new record), Japan, Russia.

\section{${ }^{1 * N o t o p l a t y l a b u s ~ p o d o l i c u s ~ c o n t e r r a n e u s ~ T e r e s h k i n, ~}$ 1993}

Notoplatylabus conterraneus Tereshkin, 1993: 477-488. Type: female; TL: Primor'ye Kray, Russia; TD: ZIN.

Material examined. Korea: 1 우, GW: Donghae-si, Samhwa-dong, Mureung Valley (M.T.), 20 Sep-2 Oct 2006, Lee JW [YNU]; 2 우 우, Donghae-si, Samhwa-dong, Mureung Valley (M.T.), 10-20 Sep 2006, Lee JW [YNU]; 2 우 우, Donghae-si, Samhwa-dong, Mureunggyegok (M.T.), 1-8 Oct 2005, Lee JW [YNU].

Diagnosis. Body length 5.7-5.8 mm, fore wing length $4.3-$ $4.5 \mathrm{~mm}$. Flagella with 31-34 segments, bristle-shape, tyloides and white band absent. Clypeus separated from face by deep furrow, strongly convex at base, its apical margin thin, with slight cavity. Scutellum raised above postscutellum. Apex and apical transverse carina of a 2 nd pleural area of propodeum with short tooth-like projection. Gastrocoeli long and thyridium broad and long.

Host. Unknown.

Distribution. Korea (new record), Russia.

${ }^{2 *}$ Oronotus ishiyamanus (Uchida, 1926)

Takanona ishiyamana Uchida, 1926: 164-165. Type: male;

TL: Ishiyama, Japan; TD: lost.

Takanoma bimaculata Uchida, 1956: 74. Type: female; TL: Hokkaido, Japan; TD: HU. Synonymized by Townes et al. (1965).

Takanoma bimaculata: Iwata, 1958: 73; 1960b: 137.

Oronotus ishiyamana: Townes, Momoi \& Townes, 1965: 420; Yu \& Horstmann, 1997: 492.

Material examined. Korea: 1 우, GG: Pyeongtaek-si, Hyeongdeok-myeon, Gisan 2-ri, Oknyeobong, 10 Sep 2006, Lee HS [YNU]; 1 ð , CN: Buyeo-gun, Sumok-ri, 7-30 Sep 2005, Lee JW [YNU]; Japan: 1 우, Hokkaido, Hyogo, Sasayamna, 29 Aug 1954, Iwata K (holotype of Takanoma bimaculata Uchida, 1956) [HU].

Diagnosis. Body length 7-8 mm. Fore wing length $4.5 \mathrm{~mm}$. Flagella with 28 segments, bristle shaped; central flagello- meres elongate. Clypeus weakly but evenly convex, simply transverse, uniformly thick. Clypeus without punctures; apical margin simple, without a median apical tubercle. Scutellum flat; lateral longitudinal carinae more or less complete to center. Gastrocoeli distinctly impressed, thyridiae placed very far from base of tergite 2 , twice as broad as distance between them.

Host. Unknown.

Distribution. Korea (new record), Japan.

3*Paraplatylabus vietnamensis Riedel, 2011

Paraplatylabus vietnamensis Riedel 2011: 1549-1572. Type: female; TL: Vietnam; TD: RIEDEL.

Material examined. Korea: 1 우, GB: Yeungnam Univ. (Malaise trap), 15 Aug-30 Sep 2006, Lee JW [YNU].

Diagnosis. Flagella with 39 segments, 1st flagellomere 6 times as long as wide. Ivory flagellar stripe is located on each 8 th to 14th flagellomeres. Face and clypeus with dark spots centrally. Hind femur slender, about 4.4 times as long as high. Superomedia area rectangular and slightly longer than wide. The hind femur dark brownish, with diffuse reddish spot in central part.

Host. Unknown.

Distribution. Korea (new record), Vietnam.

${ }^{4 *}$ Platylabus takeuchii Uchida, 1930

Platylabus takeuchii Uchida, 1930: 99. Lectotype: female; TL: Kyoto, Japan; TD: HU.

Platylabus takeuchii: Iwata, 1958: 72; 1960b: 140; Townes, Momoi \& Townes, 1965: 516; Yu \& Horstmann, 1997: 681.

Material examined. Korea: 1 우, GW: Gachilbonf, 23 Jul 1981, Kim CW [YNU]; 1 우, GB: Mt. Sobaeksan, Jukgyegugok, 4 Aug 1994, Yeo HD [YNU]; 1 우, Mt. Sobaeksan, Huibang Valley, 3 May 1997, Park SH [YNU].

Diagnosis. Body length $10 \mathrm{~mm}$. Fore wing length $7.5 \mathrm{~mm}$. Flagella with 34 segments, lanceolate from 13 segment, central flagellomeres of female elongate. Clypeus weakly but evenly convex, small and subquadrate, uniformly thick. Clypeus with punctures evenly distributed, apical margin simple, without median apical tubercle. Scutellum strongly convex, shelf-like; lateral longitudinal carinae more or less complete to center, but not reaching posterior margin. Gastrocoeli twice as broad as distance between them, and with distinctly impressed thyridium.

Host. Unknown.

Distribution. Korea (new record), Japan, India, Taiwan. 
1*Pseudoplatylabus apicalis (Uchida, 1926)

Rhexidermus apicalis Uchida, 1926: 166. Type: female; TL: Sapporo, Japan; TD: HU.

Pseudoplatylabus apicalis: Uchida, 1956: 63; Townes, 1957: 118; Iwata, 1958: 72; 1960: 137; Townes, Momoi \& Townes, 1965: 425; Yu \& Horstmann, 1997: 666.

Material examined. Korea: 1 우, GB: Gumi-si, Haepyeong wetland, 23 May 2003, Jeong JC [YNU]; 1 우, GN: Jinju-si, Gajwa-dong, 30 Sep 1987, Lee JW [YNU]; 1 우, Hapcheongun, Jeongyang-ri, 18 May 2002, Jeong JC [YNU]; Japan: 1 우, Hokkaido, Sapporo, 20 Apr 1925, Kono H (holotype of Rhexidermus apicalis Uchida, 1928) [HU].

Diagnosis. Body length $10 \mathrm{~mm}$. Fore wing length $5.5 \mathrm{~mm}$. Flagella with 30 segments, bristle shaped; central flagellomeres elongate. Clypeus flat, in same plane as lower face, simply transverse, sublenticular, centrally concave, tapering toward apex so that apical one-fifths semitranslucent. Clypeus with punctures sparsely and irregularly distributed; apical margin simple, without median apical tubercle. Scutellum moderately convex; lateral longitudinal carinae complete to posterior margin. Gastrocoeli 1.8 times as broad as distance between them, and with distinctly impressed thyridium.

Host. Unknown.

Distribution. Korea (new record), Japan.

\section{${ }^{2 *}$ Thyrateles haereticus Wesmael, 1854}

Amblyteles haereticus Wesmael, 1854: 120. Type: female; TL: sabaudia, Italy; TD: IRSNB.

Amblyteles haereticus: Taschenberg, 1870: 455; Kriechbaumer, 1888: 31; Tosquinet, 1889: 125; Dalla Torre, 1902: 816; Schmiedeknecht, 1903: 187; Berthoumieu, 1905: 123; Meyer, 1933: 306.

Ichneumon urticarum Holmgren, 1880: 23. Lectotype: female; TD: NHRS.

Ichneumon urticarum: Berthoumieu, 1894: 552; Schmiedeknecht, 1930: 206.

Coelichneumon falsificus var. urticarum: Thomson, 1893: 1905.

Amblyteles haereticus var. binotata Wesmael, 1854: 339. Lectotype: female; TD: ZSM. Homonym of Amblyteles binotatus Kriechbaumer, 1890.

Ichneumon cinctor Kriechbaumer, 1894: 48. Type: female; TD: HNHM. Homonym of Ichneumon cinctor Thunberg, 1824.

Ichneumon cinctor: Berthoumieu, 1894: 505-592; Schmiedeknecht, 1930: 334.

Ctenichneumon haereticus: Morley, 1903: 179.

Ichneumon discriminator var. pyraeneus Pic, 1914: 69. Lecto- type: male; TL: Pyrnes, France; TD: BFIC. Homonym of Ichneumon pyrenaeus Tischbein, 1882.

Coelichneumon urticarum: Meyer, 1933: 62.

Pterocormus haereticus: Townes, Momoi \& Townes, 1965: 468.

Thyreteles haereticus: Hilpert, 1992: 33; Hilpert, Hinz \& Horstmann, 1993: 181; Yu \& Horstmann, 1997: 654.

Material examined. Korea: $4 \diamond^{\nearrow}$ ऽ, GW: Mt. Seolaksan, Socheongbong, 19 Aug 1988, Lee JH; 1 ఠ, ditto, Kim HG [YNU].

Diagnosis. Body length $19 \mathrm{~mm}$. Fore wing length $15 \mathrm{~mm}$. Flagella with 43 segments. Central part of face convex. Clypeus flat, without median apical tubercle. Scutellum strongly convex and yellow; lateral longitudinal carinae absent. Lateral part of 1st tergite with transverse striae, 2nd tergite with very distinctly impressed thyridium. 2nd tergite and 3rd tergite yellow, 4th tergite with two yellow spots.

Host. [Lepidoptera] Arctiidae: Phragmatobia fuliginosa (Linnaeus); Lasiocampidae: Dendrolimus pini (Linnaeus), Penthophera morio (Linnaeus); Nymphalidae: Aglais urticae (Linnaeus), Inachis io (Linnaeus), Vanessa callirhoe (=Vanessa indica (Herbst)), Vanessa cardui (Linnaeus); Noctuidae: Noctua comes (Hübner); Sphingidae: Sphinx pinastri(Linnaeus).

Distribution. Korea (new record), Japan, Russia, Austria, Belarus, Czechoslovakia, England, Estonia, Finland, France, Germany, Hungary, Italy, Latvia, Netherlands, Norway, Poland, Romania, Russia, Spain, Sweden, Switzerland, Ukraine. Remarks. Kim (1955) recorded Ctenichneumon haereticus from Korea, but Townes et al. (1965) reported that Kim's specimens a misdetermination of Ichneumon longicrus Uchida. Therefore, our report here in is the new Korean record of this species.

\section{${ }^{3 *}$ Ulesta pieli Uchida, 1956}

Ulesta pieli Uchida, 1956: 67. Type: female; TL: Jiangwi, China; TD: HU.

Material examined. Korea: 1 우, GB: Gyongsan-si, Yeungnam Univ., 18 May 1989, Lee HU [YNU].

Diagnosis. Body length $12 \mathrm{~mm}$. Fore wing length $8 \mathrm{~mm}$. Flagella with 36 segments, central part with white band. Central part of face weakly convex. Clypeus flat, with weak median apical tubercle and rounded. Scutellum weakly convex; lateral longitudinal carinae absent. 2nd tergite with distinctly impressed thyridium.

Host. Unknown.

Distribution. Korea (new record), China.

Korean name: ${ }^{1 *}$ 납작머리방패진맵시벌 (신칭), ${ }^{2 *}$ 어리진맵시벌 (신칭), ${ }^{3 *}$ 호리뵤족진맵시벌 (신칭) 


\section{ACKNOWLEDGMENTS}

We thank Prof. Yanko Kolarov of the Faculty of Pedagogie, University of Plovdiv (Bulgaria) for providing useful comments as well as the anonymous reviewer for reviewing this manuscript. This work was supported by a grant from the National Institute of Biological Resources (NIBR), funded by the Ministry of Environment (MOE) of the Republic of Korea (NIBR No. 2013-02-001).

\section{REFERENCES}

Aubert JF, 1964. Les Ichneumonides du rivage méditerranéen français (Hym.). 7e série: Ichneumoninae, Cryptinae, Ophioninae et Mesochorinae de l'Hérault et des Bouches-duRhone. Bulletin de la Société Entomologique de France, 69:144-164.

Barron JR, 1978. Systematics of the world Eucerotinae (Hymenoptera, Ichneumonidae) Part 2. Non-nearctic species. Naturaliste Canadien, 105:327-374.

Berthoumieu V, 1894. Ichneumonides d'Europe et des pays limitrophes. Annales de la Société Entomologique de France, 63:505-592.

Berthoumieu V, 1905. Le genre "Triptognathus" Bert. (Hym.) et les variétés de "T. uniguttatus" Gr. Échange, 21:123-125, 131-133.

Brischke CGA, 1878. Die Ichneumoniden der Provinzen Westund Ost-Preussen. Schriften der Naturforschenden Gesellschaft in Danzig, 4:35-117.

Cameron P, 1903. Descriptions of twelve new genera and species of Ichneumonidae (Heresiarchini and Amblypygi) and three species of Ampulex from the Khasia Hills, India. Transactions of the Entomological Society of London, 51:219-238.

Cushman RA, 1929. Three new Ichneumonoid parasites of the rice-borer Chilo simplex (Butler). Proceedings of the Hawaiian Entomological Society, 7:243-245.

Dalla Torre CG de, 1902. Catalogus Hymenopterorum. Volumen III. Trigonalidae, Megalyridae, Stephanidae, Ichneumonidae, Agriotypidae, Evaniidae, Pelecinidae. Guilelmi Engelmann. Lipsiae, 1902:545-1141.

Förster A, 1869. Synopsis der Familien und Gattungen der Ichneumonen. Verhandlungen des Naturhistorischen Vereins der Preussischen Rheinlande und Westfalens, 25:135-221.

Gravenhorst JLC, 1829. Ichneumonologia Europaea. Pars I. Vratislaviae, pp. 1-827.

Gupta VK, 1987. The Ichneumonidae of the Indo-Australian area (Hymenoptera). Memoirs of the American Entomological Institute, No. 41. Part 2. American Entomological Institute, Gainesville, pp. 598-1210.

Heinrich GH, 1937. A list and some notes on the synonymy of the types of the subfamily Ichneumoninae Ashmead (Hym.) in the collections of the British Museum and the Hope Department of the Oxford University Museum. Annals and
Magazine of Natural History, 20:257-279.

Heinrich GH, 1951. Ichneumoniden der Steiermark (Hym.). Bonner Zoologische Beiträge, 2:235-290.

Hilpert H, 1987. Eine neue Art der Gattung Phrudus Förster (1868) (Hymenoptera, Ichneumonidae, Phrudinae). Entomofauna, 8:213-219.

Hilpert H, 1992. Zur Systematik der Gattung Ichneumon Linnaeus, 1758 in der Westpalaearktis (Hymenoptera, Ichneumonidae, Ichneumoninae). Entomofauna, Supplment, 6:1389.

Hilpert H, Hinz R, Horstmann K, 1993. Typenrevision der von Maurice Pic beschriebenen Ichneumoninae (ohne Phaeogenini) (Hymenoptera, Ichneumonidae). Spixiana, 16:173-187.

Holmgren AE, 1880. Adnotationes ad "Ichneumonologiam Suecicam.” Entomologisk Tidskrift, 1:22-32.

Humala AE, 2008. New species of Orthocentrinae (Hymenoptera: Ichneumonidae) from Finland. Entomologica Fennica, 19:94-104.

Iwata K, 1958. Ovarian eggs of 233 species of the Japanese Ichneumonidae (Hymenoptera). Acta Hymenopterologia, 1:63-74.

Iwata K, 1960a. Phenological observation on the Japanese Ichneumon flies with special reference to their fecundity (Hymenoptera). Mushi, 33:39-46.

Iwata K, 1960b. The comparative anatomy of the ovary in Hymenoptera, Part V. Ichneumonidae. Acta Hymenopterologia, 1:115-169.

Jeong JC, Cha JY, 2009. Newly recorded tribe Eurylabini of subfamily Ichneumoninae (Hymenoptera: Ichneumonidae) in Korea. Entomological Research, 39:330-333.

Jeong JC, Cha JY, Choi JK, Lee JW, 2009. Six species of the tribe Ichneumonini (Hymenoptera: Ichneumonidae) new to Korea. Korean Journal of Systematic Zoology, 25:107-119.

Jeong JC, Cha JY, Choi JK, Lee JW, 2010. Taxonomy of the genus Amblyjoppa Cameron (Hymenoptera: Ichneumonidae: Ichneumoninae) from Korea. Entomological Research, 40: 304-315.

Jeong JC, Choi JK, Lee JW, 2008. First record of the genus Probolus (Hymenoptera: Ichneumonidae: Ichneumoninae) from Korea, based on one new species and one newly recorded species. Korean Journal of Systematic Zoology, 24:99105.

Jeong JC, Lee JW, 2006. Taxonomy of Listrodromini (Hymenoptera: Ichneumonidae: Ichneumoninae) from Korea. Entomological Research, 36:127-132.

Jeong JC, Lee JW, 2008. Taxonomic review of the genus Hymenura Townes (Hymenoptera: Ichneumonidae: Ichneumoninae) in Korea. Journal of Asia Pacific Entomology, 11:1720.

Jeong JC, Lee SM, Lee JW, 2007. Redescriptions of four ichneumonine genera and species (Hymenoptera: Ichneumonidae: Ichneumoninae) new to Korea. Korean Journal of Systematic Zoology, 23:189-197.

Kasparyan DR, 1984. New species of Ichneumonidae (Hymenoptera) of the genus Euceros Grav. from the Far East. In: Sys- 
tematics of insects from the Far East. Collected scientific papers (Ed., Ler PA). Akademii Nauk. SSR, Vladivostok, 1984:78-83 (in Russian).

Kim CW, 1955. A study on the Ichneumon-flies in Korea. Commemoration These 15th Anniv. Korea Univ., pp. 423498 (in Korean with German summary).

Kim CW, 1970. Illustrated encyclopedia of fauna \& flora of Korea. Vol. 11. Insecta (III). Ministry of Education, Republic of Korea, Seoul, pp. 12-722.

Kim OS, Lee HP, 1982. Studies on the biological control of the fall webworm Hyphantria cunea Drury. Korean Journal of Entomology, 12:49-56.

Kriechbaumer J, 1888. Neue Ichneumoniden des Wiener Museums. Annalen des Naturhistorischen Hofmuseums in Wien, 3:23-36.

Kriechbaumer J, 1889. Ichneumoniden-Studien. 14. Entomologische Nachrichten, 15:142-144.

Kriechbaumer J, 1890. Ichneumoniden-Studien. 29-32. Entomologische Nachrichten, 16:181-185.

Kriechbaumer J, 1894. Ichneumonidae novae e fauna Hungarica Musaei Nationalis Hungarici. Természetrajzi Füzetek, 17: 48-60.

Kwon JM, Jeong JC, Cha JY, Lee JW, 2012. Two new records of the Tribe Phaeogenini Förster (Hymenoptera: Ichneumonidae: Ichneumoninae) from Korea. Entomological Research, 42:168-171.

Latreille PA, 1802. Histoire naturelle, générale et particulière, des Crustacés et des insectes. Tome troisième, Paris, pp. 1468 (Ichneumonidae. pp. 318-327).

Lee JW, Cha JY, 2000. Illustrated catalogue of Ichneumonidae in Korea. (1. Anomalinae, Eucerotinae, Mesochorinae, Metopiinae, Ophioninae, Paxylommatinae, Tryphoninae). Insects of Korea Series 6. Korea Research Institute of Bioscience and Biotechnology \& Center for Insect Systematics, Chuncheon, pp. 1-261.

Lee JW, Cha JY, Suh KI, 1992. A systematic study of the Ichneumonidae (Hymenoptera) from Korea XIV. Genus Euceros (Eucerotinae). Entomological Research Bulletin (Korea), 18:25-30.

Meyer NF, 1933. Keys to parasitic Hymenoptera (family Ichneumonidae) of the USSR and adjacent countries. Vol. 1. Introduction and Ichneumoninae. Zoological Institute of the Academy of Sciences of the USSR, 9:1-458.

Morley C, 1903. Ichneumonologia Britannica. The Ichneumons of Great Britain. Ichneumoninae. Plymouth, pp. 1-315.

Morley C, 1907. Ichneumonologia Britannica. II. The Ichneumons of Great Britain. Cryptinae. Plymouth, pp. 1-351.

Pic M, 1914. Diagnoses de divers Ichneumoniens. Échange, 30:69-70.

Riedel M, 2011. Contribution to the Ichneumoninae (Hymenoptera, Ichneumonidae) of Southeastern Asia: 1. Tribes Clypeodromini, Listrodromini, Goedartini, Compsophorini, and Platylabini. Linzer Biologische Beitraege, 43:1549-1572.

Roman A, 1904. Några svenska Ichneumonid-fynd. Entomologisk Tidskrift, 25:115-120.
Schmiedeknecht O, 1903. Opuscula Ichneumonologica. I. Band. (Fasc. II-IV.) Bestimmungstabelle der paläarktischen Arten der Gattung Ichneumon. Bestimmungstabelle und Beschreibungen weiter Gattungen der Ichneumoninae. Blankenburg in Thüringen, pp. 81-320.

Schmiedeknecht O, 1910. Opuscula Ichneumonologica. IV. Band. (Fasc. XXIV-XXVI.) Ophioninae. Blankenburg in Thüringen, pp. 1841-2080.

Schmiedeknecht O, 1928. Opuscula Ichneumonologica. Supplement-Band. Neubearbeitungen. Fasc. I-IV. Genus Ichneumon L. Blankenburg in Thüringen, pp. 1-272.

Schmiedeknecht O, 1930. Die Hymenoptera Nord- und Mitteleuropas. Verlag von Gustav Fischer, Jena, pp. 1-1062.

Smith F, 1874. Description of new species of Tenthredinidae, Ichneumonidae, Chrysididae, Formicidae etc. of Japan. Transactions of the Entomological Society of London, 1874: 373-409.

Strobl G, 1901. Ichneumoniden Steiermarks (und der Nachbarländer). Mitteilungen Naturwissenschaftlichen Vereines für Steiermark, Graz, 37:132-257.

Takahashi H, 1995. Ichneumonoidea from Hachijo-jima Island, Izu Islands. Gensei, 67:7-8.

Taschenberg EL, 1870. Die Arten der Gattung Ichneumon Gr. mit linealen oder lineal-elliptischen Luftlöchern des Hinterrückens. Zeitschrift für die Gesammten Naturwissenschaften, 36:209-272.

Taschenberg EL, 1871. Einige neue südeuropäische Hymenoptera. Zeitschrift für die Gesammten Naturwissenschaften, 38:305-311.

Tereshkin A, 1993. New and little known species of Ichneumonidae Stenopneusticae of the genera Ulesta Cameron, 1903, Notoplatylabus Heinrich, 1934, and Neischnus Heinrich, 1952 (Hymenoptera, Ichneumonidae). Entomofauna, 14: 477-488.

Thomson CG, 1888. XXXVIII. Försök till gruppering af slägtet Plectiscus (Grav.). Opuscula Entomologica. Lund, 12:12661318.

Thomson CG, 1893. XLVIII. Anmärkningar öfver Ichneumoner särskildt med hänsyn till några af A.E. Holmgrens typer. Opuscula Entomologica Lund, 18:1889-1967.

Thunberg CP, 1824. Ichneumonidea, Insecta Hymenoptera illustrata. Mémoires de l'Académie Imperiale des Sciences de St. Petersbourg, 9:285-368.

Tischbein PFL, 1882. Zusätze und Bemerkungen zu der Übersicht der europäischen Arten des Genus Ichneumon Gr. Stettiner Entomologische Zeitung, 43:475-486.

Tosquinet J, 1889. Documents pour servir à la monographie des Ichneumonides de la Russie d'Asie. Annales de la Société Entomologique de Belgique, 33:125-148.

Townes HK, 1957. A review of the generic names proposed for old world Ichneumonids, the types of whose genotypes are in Japan, Formosa or North America. Proceedings of the Entomological Society of Washington, 59:100-120.

Townes HK, 1958. The application of the name Plectiscus (Hymenoptera, Ichneumonidae). Proceedings of the Ento- 
mological Society of Washington, 60:221.

Townes HK, 1969. The genera of Ichneumonidae, Part 1. Memoirs of the American Entomological Institute, No. 11. American Entomological Institute, Ann Arbor, MI, pp. 300.

Townes HK, Momoi S, Townes M, 1965. A catalogue and reclassification of the eastern Palearctic Ichneumonidae. Memoirs of the American Entomological Institute, No. 5. American Entomological Institute, Ann Arbor, MI, pp. 1661.

Townes HK, Townes M, 1949. A revision of the genera and of the American species of Tryphoninae Part I. Annals of the Entomological Society of America, 42:321-395.

Townes HK, Townes M, Gupta VK, 1961. A catalogue and reclassification of the Indo-Australian Ichneumonidae. Memoirs of the American Entomological Institute, No. 1. American Entomological Institute, Ann Arbor, MI, pp. 1522.

Uchida T, 1925a. Einige neue Ichneumoninen-Arten aus Formosa. Transactions of the Natural History Society of Formosa. Taihoku, 15:239-249.

Uchida T, 1925b. Das systematische Studium über die Tribus Joppini der Unterfamilie Ichneumoninae von Japan. Dobutsugaku Zasshi [Zoological Magazine], 1925:443-457 (in Japanese with German summary).

Uchida T, 1926. Erster Beitrag zur Ichneumoniden [-Fauna] Japans. Journal of the Faculty of Agriculture, Hokkaido Imperial University, 18:43-173.

Uchida T, 1927. Über die japanische Ichneumonidengattung Eupalamus Wesm. Insecta Matsumurana, 2:202-205.

Uchida T, 1930. Beschreibung einer neuen Gattung und einiger neuen Ichneumoniden-Arten aus Japan. Insecta Matsumurana, 5:94-100.

Uchida T, 1932. H. Sauter's Formosa-Ausbeute. Ichneumonidae (Hym.). Journal of the Faculty of Agriculture, Hokkaido Imperial University, 33:133-222.

Uchida T, 1935. Zur Ichneumonidenfauna von Tosa (I.) Subfam. Ichneumoninae. Insecta Matsumurana, 10:6-33.

Uchida T, 1936. Einige Hymenopteren aus dem Berg Daisetsu. Biogeographica. Transactions of the Biogeographical Society of Japan, 1:63-74.

Uchida T, 1939. Die smithschen Typen der japanischen Ichneumoniden. Insecta Matsumurana, 14:27-36.

Uchida T, 1953. Die Ichneumoniden (Hymenoptera). Die Insek- tenfauna aus dem Berg Ishizuchi und dem Tal Omogo. Transactions of the Shikoku Entomological Society, 3:126134.

Uchida T, 1955. Neue oder wenig bekannte Schmarotzer der Nadelholz-Blattwespen nebst einem neuen sekundären Schmarotzer. Insecta Matsumurana, 19:1-8.

Uchida T, 1956. Neue oder bisher unbekannte Ichneumoniden aus Japan und seinen Umgegenden (I). Insecta Matsumurana, 20:57-76.

Uchida T, 1958. Systematische Übersicht der Euceros-Arten Japans (Hym., Ichneumonidae). Mushi, 32:129-133.

Viereck HL, 1919. Additions and corrections to a list of families and subfamilies of the Ichneumon-flies in the superfamily Ichneumonoidea. Proceedings of the Biological Society of Washington, 32:48-198.

Wahl DB, Sharkey MJ, 1993. Superfamily Ichneumonoidea. In: Hymenoptera of the world: an identification guide to families. Agriculture Canada Publication, 1894E (Eds., Goulet H, Huber JT). Agriculture Canada Publication, Ottawa, pp. 358-509.

Walker F, 1874. Descriptions of some Japanese Hymenoptera. Cistula Entomologica, 1:301-310.

Wesmael C, 1854. Ichneumones Amblypygi Europaei. Ichneumones Amblypygi. Bulletin de l'Académie Royale des Sciences, Belgique, 21:77-142.

Wesmael C, 1857. Ichneumonologica otia. Bulletin de l'Académie Royale des Sciences, Belgique, 26:355-426.

Yu DS, Horstmann K, 1997. A catalogue of world Ichneumonidae (Hymenoptera). Memoirs of the American Entomological Institute. Vol. 58. American Entomological Institute, Gainesville, pp. 1-1558.

Yu DS, Van Achterberg C, Horstmann K, 2005. World Ichneumonoidea 2004. Taxonomy, biology, morphology and distribution [Ichneumonidae]. Taxapad 2005, Interactive catalogue on CD-ROM, Vancouver, Canada.

Yu DS, Van Achterberg C, Horstmann K, 2012. Taxapad 2012, Ichneumonoidea 2011. Database on flash-drive [Internet]. Dicky Sick Ki Yu, Ottawa, Accessed 1 Jan 2014, <http:// www.taxapad.com>. 